\title{
APPROXIMATE EVALUATION OF A CLASS OF WIENER INTEGRALS ${ }^{1}$
}

\section{J. YEH}

1. It is well known that for every $\alpha \in\left(0, \frac{1}{2}\right)$ almost every element $x$ of the Wiener space $C_{w}$ consisting of all the real valued functions $x(t), t \in[0,1]$, with $x(0)=0$ satisfies the Hölder condition $\left|x\left(t^{\prime}\right)-x\left(t^{\prime \prime}\right)\right|$ $\leqq h\left|t^{\prime}-t^{\prime \prime}\right| \alpha$ for some $h>0$ which depends on $x$ and $\alpha$. Let $\phi_{\alpha}[x]$ be the infimum of all such $h>0$ for fixed $x$ and $\alpha$. In [7] we showed that for every $\alpha \in\left(0, \frac{1}{6}\right)$ the functional $\phi_{\alpha}^{p}[x]$ is Wiener integrable for every $p \geqq 0$ and in fact

$$
\begin{aligned}
\int_{C_{w}} \phi_{\alpha}^{p}[x] d_{w} x \leqq & 2^{p}\left(1-2^{-\alpha}\right)^{-p} \\
& +\left(\frac{2}{\pi}\right)^{1 / 2} 2^{3 \alpha-1 / 2}\left(1-2^{3 \alpha-1 / 2}\right)^{-1} 2^{p}\left(1-2^{-\alpha}\right)^{-p} \\
& \cdot \sum_{m=1}^{\infty}(m+1)^{p} m^{-(m+1)}<\infty .
\end{aligned}
$$

We then applied this result to estimate errors in approximating Wiener integrals of a class of functionals by Lebesgue integrals in Euclidean spaces. In the present paper we apply this method to yet another class of Wiener integrals. Our result is the following:

THEOREM. Let $f(t)$ be real valued for $t \in[0,1]$ and satisfy

$$
\left|f\left(t^{\prime}\right)-f\left(t^{\prime \prime}\right)\right| \leqq C\left|t^{\prime}-t^{\prime \prime}\right|^{\gamma}
$$

where $C, \gamma>0$ and let $A=\max _{[0,1]}|f(t)|$. Let $g(u)$ be real valued for all real $u$ and satisfy

$$
\left|g\left(u^{\prime}\right)-g\left(u^{\prime \prime}\right)\right| \leqq B\left|u^{\prime}-u^{\prime \prime}\right|
$$

with $B>0$. Then the functionals $F[x]$ and $F_{n}[x]$ defined on $C_{w}$ by

$$
\begin{aligned}
F[x] & =g\left\{\int_{0}^{1} f(t) x^{2}(t) d t\right\} \\
F_{n}[x] & =g\left\{\frac{1}{n} \sum_{k=1}^{n} f\left(\frac{k}{n}\right) x^{2}\left(\frac{k}{n}\right)\right\}, \quad n=1,2, \cdots
\end{aligned}
$$

Received by the editors October 18, 1967.

1 This research was supported in part by National Science Foundation Grant GP-5436. 
are Wiener measurable, and for $\alpha \in\left(0, \frac{1}{6}\right)$

$$
\begin{aligned}
\left|\int_{C_{w}} F[x] d_{w} x-\int_{C_{w}} F_{n}[x] d_{w} x\right| \\
\leqq \frac{2 B C}{(\gamma+1) n^{\gamma}}+\frac{2^{3 / 2} A B}{(\alpha+1) n^{\alpha}}\left\{\int_{C_{w}} \phi_{\alpha}^{2}[x] d_{w} x\right\}^{1 / 2} .
\end{aligned}
$$

The Wiener integral $\int_{C_{w}} F_{n}[x] d_{w} x$ which approximates $\int_{C_{w}} F[x] d_{w} x$ can be evaluated as a Lebesgue integral in the $n$-dimensional Euclidean space according to the well-known fact that if $G\left(\xi_{1}, \cdots, \xi_{n}\right)$ is a Lebesgue measurable function defined on the $n$-dimensional Euclidean space and $0=t_{0}<t_{1}<\cdots<t_{n} \leqq 1$ then $G\left[x\left(t_{1}\right), \cdots, x\left(t_{n}\right)\right]$ is Wiener measurable and furthermore

$$
\begin{aligned}
& \int_{C_{w}} G\left[x\left(t_{1}\right), \cdots, x\left(t_{n}\right)\right] d_{w} x=\left\{(2 \pi)^{n} \sum_{k=1}^{n}\left(t_{k}-t_{k-1}\right)\right\}^{-1 / 2} \cdot \\
& \int_{-\infty}^{\infty} \cdots \int_{-\infty}^{\infty} G\left(\xi_{1}, \cdots, \xi_{n}\right) \exp \left\{-\sum_{k=1}^{n} \frac{\left(\xi_{k}-\xi_{k-1}\right)^{2}}{2\left(t_{k}-t_{k-1}\right)}\right\} d \xi_{1} \cdots d \xi_{n}
\end{aligned}
$$

in the sense that the existence of one side implies that of the other and the equality of the two. Thus for our $F_{n}[x]$ we have

$$
\begin{aligned}
\int_{C_{w}} F_{n}[x] d x_{w} x=(2 \pi)^{-n / 2} n^{n / 2} \int_{-\infty}^{\infty} & \cdots \int_{-\infty}^{\infty} g\left\{\frac{1}{n} \sum_{k=1}^{n} f\left(\frac{k}{n}\right) \xi_{k}^{2}\right\} \\
& \exp \left\{-\frac{n}{2} \sum_{k=1}^{n}\left(\xi_{k}-\xi_{k-1}\right)^{2}\right\} d \xi_{1} \cdots d \xi_{n} .
\end{aligned}
$$

2. In proving our theorem we need to estimate the Wiener integral

$$
\int_{C_{w}}\||| x \mid\|{ }^{2} d_{w} x \quad \text { where }\||x|\|=\max _{[0,1]}|x(t)| .
$$

In [2] P. Erdös and M. Kac showed that if $X_{1}, X_{2}, X_{3}, \cdots$ are independent identically distributed random variables each having mean value 0 and standard deviation 1 and if $s_{k}=X_{1}+X_{2}+\cdots+X_{k}$ then

$$
\lim _{n \rightarrow \infty} \operatorname{prob}\left\{\left\{\max \left(s_{1}, s_{2}, \cdots, s_{n}\right)<\alpha n^{1 / 2}\right\}=\sigma(\alpha)\right.
$$

where $\sigma(\alpha)=0$ for $\alpha \leqq 0$ and

$$
\sigma(\alpha)=\left(\frac{2}{\pi}\right)^{1 / 2} \int_{0}^{\alpha} \exp \left\{-\frac{u^{2}}{2}\right\} d u \quad \text { for } \alpha \geqq 0 .
$$


From this follows immediately

Theorem A. Let $f(u)$ be measurable on $[0, \infty)$. Then if either of the following integrals exists, both exist and they are equal:

$$
\int_{C_{w}} f[\max x(t)] d_{w} x=\left(\frac{2}{\pi}\right)^{1 / 2} \int_{0}^{\infty} f(u) \exp \left\{-\frac{u^{2}}{2}\right\} d u .
$$

Corollary B. Let $f(u)$ be measurable and nonnegative on $[0, \infty)$. Then

$$
\begin{aligned}
\left(\frac{2}{\pi}\right)^{1 / 2} \int_{0}^{\infty} f(u) \exp \left\{-\frac{u^{2}}{2}\right\} d u \leqq & \int_{C_{w}} f(\|x\|||) d_{w} x \\
& \leqq 2\left(\frac{2}{\pi}\right)^{1 / 2} \int_{0}^{\infty} f(u) \exp \left\{-\frac{u^{2}}{2}\right\} d u
\end{aligned}
$$

where the left-hand inequality holds provided that $f(u)$ is monotonically increasing.

Corollary B follows from Theorem A and the observation that $|u|=\max \{u,-u\}$ so that

$$
\||x|\|=\max \left\{\max _{[0,1]} x(t), \max _{[0,1]}[-x(t)]\right\}
$$

and hence if $f(u) \geqq 0$ (and for the left-hand inequality, $f$ is also monotonically increasing),

$$
f[\max x(t)] \leqq f(|\|x \mid\|) \leqq f[\max x(t)]+f[\max [-x(t)]]
$$

so that finally

$$
\begin{aligned}
\int_{C_{w}} f[\max x(t)] d_{w} x & \leqq \int_{C_{w}} f(\|x \mid\|) d_{w} x \\
& \leqq \int_{C_{w}} f[\max x(t)] d_{w} x+\int_{C_{w}} f[\max [-x(t)]] d_{w} x \\
& =2 \int_{C_{w}} f[\max x(t)] d_{w} x .
\end{aligned}
$$

3. Proof of the theorem. From the continuity of $g(u)$, the function

$$
g\left\{\frac{1}{n} \sum_{k=1}^{n} f\left(\frac{k}{n}\right) \xi_{k}^{2}\right\}
$$

is continuous on the $n$-dimensional Euclidean space with elements $\left(\xi_{1}, \cdots, \xi_{n}\right)$ and hence $F_{n}[x]$ as defined by $(4)$ is Wiener measurable. 
Since $x(t)$ and $f(t)$ are continuous the Riemann sum in (4) converges to the Riemann integral in (3) as $n \rightarrow \infty$. Then from the continuity of $g(u), \lim _{n \rightarrow \infty} F_{n}[x]=F[x]$ for every $x \in C_{w}$. This establishes the Wiener measurability of $F[x]$. Now

$$
\begin{aligned}
\mid \int_{0}^{1} f(t) x^{2}(t) d t-\frac{1}{n} & \sum_{k=1}^{n} f\left(\frac{k}{n}\right) x^{2}\left(\frac{k}{n}\right) \mid \\
\leqq & \sum_{k=1}^{n} \int_{(k-1) / n}^{k / n}\left|f(t) x^{2}(t)-f\left(\frac{k}{n}\right) x^{2}\left(\frac{k}{n}\right)\right| d t \\
\leqq & \sum_{k=1}^{n} \int_{(k-1) / n}^{k / n}\left|f(t) x^{2}(t)-f\left(\frac{k}{n}\right) x^{2}(t)\right| d t \\
& +\sum_{k=1}^{n} \int_{(k-1) / n}^{k / n}\left|f\left(\frac{k}{n}\right) x^{2}(t)-f\left(\frac{k}{n}\right) x^{2}\left(\frac{k}{n}\right)\right| d t \\
\leqq & \|\left. x||\right|^{2} \sum_{k=1}^{n} \int_{(k-1) / n}^{k / n}\left|f(t)-f\left(\frac{k}{n}\right)\right| d t \\
& +2 A\left|\left\|x\left|\| \sum_{k=1}^{n} \int_{(k-1) / n}^{k / n}\right| x(t)-x\left(\frac{k}{n}\right) \mid d t\right.\right. \\
\leqq & C|\| x|||^{2} \sum_{k=1}^{n} \int_{(k-1) / n}^{k / n}\left(\frac{k}{n}-t\right)^{\gamma} d t \\
& +2 A\left|\|x \mid\| \sum_{k=1}^{n} \int_{(k-1) / n}^{k / n} \phi_{\alpha}[x]\left(\frac{k}{n}-t\right)^{\alpha} d t\right. \\
\leqq & \frac{C}{(\gamma+1) n^{\gamma}}\left|\left\|\left.x||\right|^{2}+\frac{2 A}{(\alpha+1) n^{\alpha}}|\|x\|| \mid \phi_{\alpha}[x] .\right.\right.
\end{aligned}
$$

Thus by (2)

$$
\begin{aligned}
\left|F[x]-F_{n}[x]\right| & =\left|g\left\{\int_{0}^{1} f(t) x^{2}(t) d t\right\}-g\left\{\frac{1}{n} \sum_{k=1}^{n} f\left(\frac{k}{n}\right) x^{2}\left(\frac{k}{n}\right)\right\}\right| \\
& \leqq B\left|\int_{0}^{1} f(t) x^{2}(t) d t-\frac{1}{n} \sum_{k=1}^{n} f\left(\frac{k}{n}\right) x^{2}\left(\frac{k}{n}\right)\right| \\
& \leqq \frac{B C}{(\gamma+1) n^{\gamma}}\left\|x \left|\left\|^{2}+\frac{2 A B}{(\alpha+1) n^{\alpha}}|| x \mid\right\| \phi_{\alpha}[x]\right.\right.
\end{aligned}
$$

and hence 


$$
\begin{aligned}
\mid \int_{C_{w}} F[x] d_{w} x- & \int_{C_{w}} F_{n}[x] d_{w} x \mid \leqq \frac{B C}{(\gamma+1) n^{\gamma}} \int_{C_{w}}\|x\|^{2} d_{w} x \\
& +\frac{2 A B}{(\alpha+1) n^{\alpha}}\left\{\int_{C_{w}}\|\| x \|^{2} d_{w} x\right\}^{1 / 2}\left\{\int_{C_{w}} \phi_{\alpha}^{2}[x] d_{w} x\right\}^{1 / 2} .
\end{aligned}
$$

Finally according to Corollary B

$$
\int_{C_{\infty}}\|x\|^{2} d_{w} x \leqq 2\left(\frac{2}{\pi}\right)^{1 / 2} \int_{0}^{\infty} u^{2} \exp \left\{-\frac{u^{2}}{2}\right\} d u=2 .
$$

This completes the proof of (5).

\section{BIBLIOGRAPHY}

1. R. H. Cameron, A Simpson's rule for the numerical evaluation of Wiener's integrals in function space, Duke Math. J. 18 (1951), 111-130.

2. P. Erdös and M. Kac, On certain limit theorems of the theory of probability, Bull. Amer. Math. Soc. 52 (1946), 292-302.

3. I. M. Gelfand and A. M. Jaglom, Integration in function spaces and its application in quantum physics, Uspekhi Mat. Nauk 11 (1956), no. 1 (67), 77-114. (Russian)

4. M. Kac, On some connections between probability theory and differential and integral equations, Proc. 2nd Berkeley Sympos. in Mathematical Statistics and Probability, 1951, pp. 189-215.

5. I. M. Koval'cik, Wiener integral, Uspekhi Mat. Nauk 18 (1963), no. 1 (109), 97-134. (Russian)

6. N. Wiener, Generalized harmonic analysis, Acta Math. 55 (1930), 117-258.

7. J. Yeh, Minimal coefficients in Hölder conditions and approximate evaluation of Wiener integrals (to appear).

University of California, Irvine 